\title{
Slow Light Beam Splitter
}

\section{Citation}

Xiao, Yanhong, Mason Klein, Michael Hohensee, Liang Jiang, David F. Phillips, Mikhail D. Lukin, and Ronald L. Walsworth. 2008. “Slow Light Beam Splitter." Physical Review Letters 101 (4). https://doi.org/10.1103/physrevlett.101.043601.

\section{Permanent link}

http://nrs.harvard.edu/urn-3:HUL.InstRepos:41461275

\section{Terms of Use}

This article was downloaded from Harvard University's DASH repository, and is made available under the terms and conditions applicable to Other Posted Material, as set forth at http:// nrs.harvard.edu/urn-3:HUL.InstRepos:dash.current.terms-of-use\#LAA

\section{Share Your Story}

The Harvard community has made this article openly available.

Please share how this access benefits you. Submit a story.

\section{Accessibility}




\title{
Slow Light Beam Splitter
}

\author{
Yanhong Xiao, ${ }^{1}$ Mason Klein, ${ }^{1,2}$ Michael Hohensee, ${ }^{1,2}$ Liang Jiang, ${ }^{2}$ David F. Phillips, ${ }^{1}$ \\ Mikhail D. Lukin, ${ }^{2}$ and Ronald L. Walsworth ${ }^{1,2}$ \\ ${ }^{1}$ Harvard-Smithsonian Center for Astrophysics, Cambridge, Massachusetts, 02138 USA \\ ${ }^{2}$ Department of Physics, Harvard University, Cambridge, Massachusetts, 02138 USA
}

(Received 19 February 2008; published 25 July 2008)

\begin{abstract}
We demonstrate a slow light beam splitter using rapid coherence transport in a wall-coated atomic vapor cell. We show that particles undergoing random and undirected classical motion can mediate coherent interactions between two or more optical modes. Coherence, written into atoms via electromagnetically induced transparency using an input optical signal at one transverse position, spreads out via ballistic atomic motion, is preserved by an antirelaxation wall coating, and is then retrieved in outgoing slow light signals in both the input channel and a spatially-separated second channel. The splitting ratio between the two output channels can be tuned by adjusting the laser power. The slow light beam splitter may improve quantum repeater performance and be useful as an all-optical dynamically reconfigurable router.
\end{abstract}

\section{DOI: 10.1103/PhysRevLett.101.043601}

In this Letter, we report the demonstration of a dynamically controllable beam splitter using slow light and rapid transport of atomic coherence in a wall-coated atomic vapor cell. In warm atomic ensembles, the internal degrees of freedom (spin states) of atoms are effectively decoupled from their external (motional) degrees of freedom. We exploit this decoupling to split an input optical signal into two spatially-separated output signals by (i) writing a collective internal state coherence into an atomic ensemble at one transverse position using electromagnetically induced transparency (EIT), (ii) letting atomic motion distribute this coherence throughout the cell, and (iii) reading out two slow light signals at both the original location and a second transverse location set by a second control field (see Figs. 1 and 2). Transport of coherence between channels at rates faster than the rate of change of the internal atomic state distinguishes this scheme from previous experimental work [1] and other EIT proposals related to light splitting [2]. A key result of this work is the demonstration that even particles undergoing random and undirected classical motion can mediate coherent interactions between two or more quantum states. This work is relevant to a range of fields not only for its potential uses, but also as a demonstration of a widely applicable concept. The slow light beam splitter may be useful in both classical and quantum information processing, e.g., as an all-optical dynamically reconfigurable router and for the efficient production of entanglement for quantum repeaters [3].

We realize rapid transport of coherence using a wallcoated atomic vapor cell with no buffer gas. Coating glass cell walls with coherence-preserving material such as paraffin [4] enables atoms to undergo thousands of wall collisions without destroying their internal state, thus enhancing the coherence lifetime and narrowing ground state linewidths. Coated cells have been used successfully in atomic clocks [5], magnetometers [6], slow light [7,8], and squeezing and entanglement generation [9]. EIT results from optical pumping of an atomic ensemble into a non-
PACS numbers: 42.50.Gy, 03.67.Hk, 32.70.Jz, 42.50.Md

interacting collective "dark state" for two optical fields (the signal and control fields) in two-photon Raman resonance with a pair of long-lived ground states of the atomic system [10]. Near an EIT transmission resonance, there is a correspondent steep dispersion which causes a weak signal pulse to propagate with reduced group velocity [7,11-13].

EIT line shapes in coated cells exhibit a dual structure: a narrow peak on top of a broad pedestal [8] [e.g., see Fig. 3(a) below]. The broad pedestal arises from the single pass, transit-time-limited interaction of atoms moving ballistically through the laser beam. The narrow peak forms in the weak laser intensity limit in which atoms undergo little evolution of their internal state between velocity-changing wall collisions (the Dicke regime [14]). The atoms experience a Doppler-free, averaged laser field with long interaction time, resulting in a narrow EIT linewidth determined by decoherence from wall collisions, spinexchange, magnetic field gradients, etc. A quantitative description of coated-cell EIT is provided by the repeated Ramsey sequence model $[15,16]$. Slow light pulses with bandwidths comparable to the narrow peak linewidth propagate in the presence of rapid transport of coherence throughout the cell, thereby enabling slow light beam

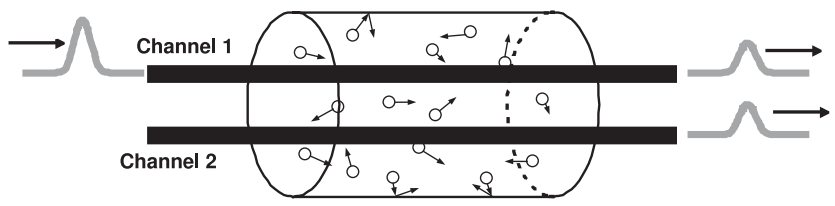

FIG. 1. Schematic of slow light beam splitter operation. An optical signal pulse enters an atomic EIT medium in Channel 1 together with a strong optical control field. Rapid atomic motion in a wall-coated cell with no buffer gas distributes atomic coherence throughout the medium. Output optical signal pulses leave the medium in both Channel 1 and a spatially-separated Channel 2 defined by the position of a second control field. The propagation direction of the two control fields, and hence the two output signal pulses, need not be the same. 


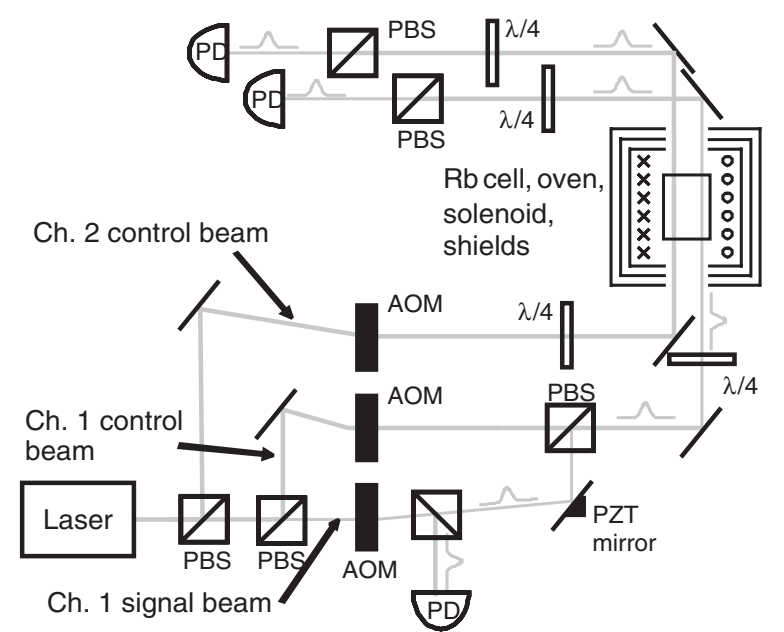

FIG. 2. Schematic of the apparatus used in the coated cell slow light beam splitter experiments. (See text for details.)

splitter operation. The properties of the slow light beam splitter can be controlled by balancing interaction time, time outside the laser beam, and power broadening by adjusting the laser intensity and beam size.

We used the experimental apparatus shown schematically in Fig. 2 to demonstrate the slow light beam splitter. An external cavity diode laser operating at the ${ }^{87} \mathrm{Rb} D_{1}$ line $(795 \mathrm{~nm})$ provided the light for the signal and control fields. The laser beam was spatially split into three beams using half-wave plates (not shown) and polarizing beam splitters (PBS): orthogonally polarized input signal and control beams were recombined and sent into Channel 1 (Ch. 1), while the second control beam was sent into Channel 2 (Ch. 2) at a different transverse location of the vapor cell (see Figs. 1 and 2). The signal and control fields formed an EIT $\Lambda$-system between two ${ }^{87} \mathrm{Rb}$ ground electronic state Zeeman sublevels $\left|F=2, m_{F}=0\right\rangle$ and $\left|F=2, m_{F}=2\right\rangle$, and an excited state $\left|F^{\prime}=1, m_{F^{\prime}}=1\right\rangle$. Acousto-optic modulators (AOM) provided intensity control for the optical fields. The two AOMs controlling the signal and control fields of Ch. 1 were driven from the same oscillator to stabilize their phase relationship. The input signal beam was reflected off a PZT-controlled mirror to adjust its path length and thus its phase relative to the Ch. 1 control field. All three laser beams were $3 \mathrm{~mm}$ in diameter, and the transverse separation between the centers of the two channels was $8 \mathrm{~mm}$. Quarter-wave plates $(\lambda / 4)$ located before and after the cell converted all fields from linear to circular polarization and back. At the output, PBS cubes transmitted only the signal field light in each channel to the photodetectors (PD). A Pyrex vapor cell $2.5 \mathrm{~cm}$ in diameter and $5 \mathrm{~cm}$ in length was coated evaporatively with tetracontane $\left(\mathrm{C}_{40} \mathrm{H}_{82}\right)$ [8] and filled with several $\mathrm{mg}$ of natural abundance rubidium. The vapor cell was heated to about $65^{\circ} \mathrm{C}$ by a blown air plastic oven, housed inside three layers of high-permeability magnetic shielding to screen out external magnetic fields. A solenoid between
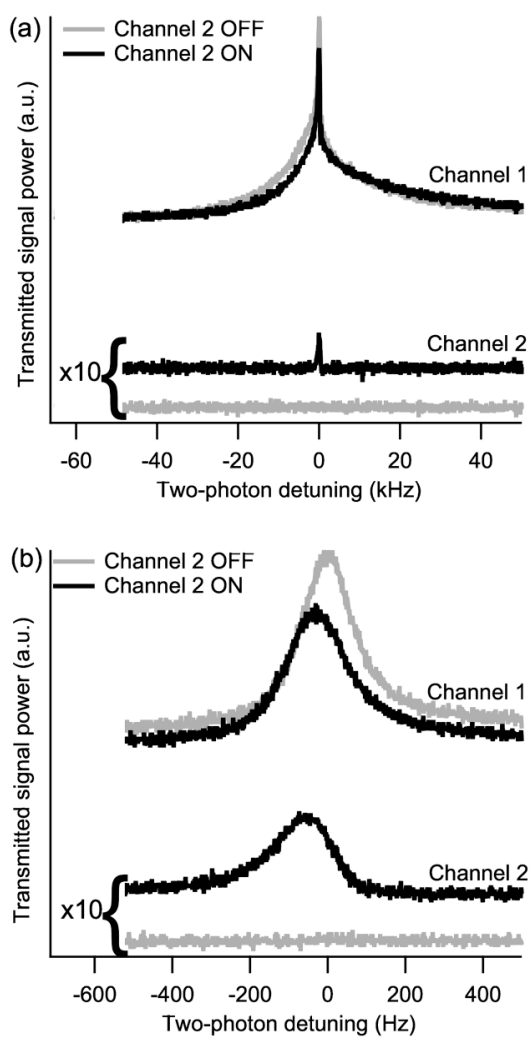

FIG. 3. Example measurements of EIT spectra in Chs. 1 and 2, demonstrating steady-state beam splitter operation. (a) Both the broad pedestal and narrow peak of the coated-cell EIT line shape are visible in the Ch. 1 output with large Ch. 1 input control power of $600 \mu \mathrm{W}$. Only the narrow EIT structure is observed in Ch. 2 with the Ch. 2 control field on because coherence associated with the broad pedestal dephases before it is transferred from Ch. 1 to Ch. 2. Ch. 1 input signal power of $9 \mu \mathrm{W}$; Ch. 2 input control power of $150 \mu \mathrm{W}$. (b) Only the narrow EIT line shape structure is visible with weak $\mathrm{Ch}$. 1 control power of $270 \mu \mathrm{W}$ and other parameters as in (a). Note: frequency range $\simeq 100$-times smaller than in (a). (For both plots: linear scales for power; Ch. 2 background from control field leakage due to finite extinction ratio of PBS.)

the oven and magnetic shields generated a uniform magnetic field used to adjust the two-photon detuning. EIT line shapes were measured by sweeping the magnetic field while observing the output $\mathrm{cw}$ signal light in each channel. Slow light pulses were shaped using an AOM, with a small part picked off as a reference for measuring pulse delay through the atomic medium.

To demonstrate steady-state operation of the slow light beam splitter, constant intensity signal and control fields were sent into Ch. 1, for the two cases of the Ch. 2 control field on and off. Figure 3(a) shows the detected output signal in both channels as the two-photon detuning was swept slowly. A strong Ch. 1 control field was used in this example so that both the broad and narrow EIT line shape features described above could be observed [8]. With no control field in Ch. 2, EIT was only visible in Ch. 1; with the Ch. 2 control field on, an EIT signal also appeared in 
Ch. 2, and the medium acted as a steady-state beam splitter. Note that the broad pedestal of the coated-cell EIT line shape does not transfer into Ch. 2, because coherence associated with the broad structure dephases on a time scale short compared to the time to reach the second channel. The signal transfer efficiency between channels is limited by the finite fraction of atoms that repeatedly cross the Ch. 2 laser beam with sufficiently small longitudinal velocity to establish good coupling to the Ch. 2 control field during the finite coherence time. With a larger filling fraction for the laser beams in the cell and/or improved wall coatings, such that decoherence is reduced by $\sim 100$, we estimate that the signal transfer efficiency would be of order unity. We also observed a decrease in the Ch. 1 EIT amplitude when the Ch. 2 control field is on, due to partial readout in $\mathrm{Ch} .2$ of the finite atomic coherence in the cell and increased optical pumping, and also a shift of the Ch. 1 EIT resonance center frequency when the Ch. 2 control field is on, due to an increase in the ac Stark shift of the EIT resonance, proportional to the total control field power in the cell (in both Ch. 1 and 2). Figure 3(b) illustrates this behavior for relatively weak control fields so that the EIT line shape is dominated by the narrow structure [8].

To demonstrate pulsed operation of the slow light beam splitter, a constant control field and a pulsed input signal field were sent into Ch. 1, again for the two cases of Ch. 2 control field on and off. Figure 4(a) shows examples of output signal pulses observed in both channels. The experimental parameters were selected to emphasize the role of effusive transport of atomic coherence between channels. Thus, the larger pulse delay observed in Ch. 2 is primarily a result of the time required for atoms to propagate effusively from Ch. 1 to Ch. 2, with internal state coherence preserved by the wall coating. An output signal pulse in Ch. 2 is not present with either control field turned off, because the output pulse in Ch. 2 comes from the transferred coherence generated in $\mathrm{Ch}$. 1. The slow light beam splitter is also tunable: the area (i.e., energy) of the signal pulse transferred from Ch. 1 to $\mathrm{Ch} .2$ can be varied by changing the laser power in either control field. Figure 4(b) shows the Ch. 2 signal pulse area as a function of Ch. 2 control field power for three Ch. 1 control field powers. With increasing Ch. 2 control power, the generated signal pulse area first grows because entering coherence is more efficiently read out, and then drops when the Ch. 2 control field performs significant optical pumping of atoms in a single beam crossing, destroying the atomic coherence and leading to increased absorption. The optimal Ch. 2 control power also depends on the $\mathrm{Ch} .1$ control power: for a weak Ch. 1 control field, even low Ch. 2 control power will optically pump atoms out of the dark state; for sufficiently high Ch. 1 control power, the benefit of more efficient Ch. 2 readout saturates (when the atom-light interaction is strong enough to read out atomic coherence in a single trip through the Ch. 2 beam), and the optimal Ch. 2 control power becomes independent of the specific Ch. 1 power.
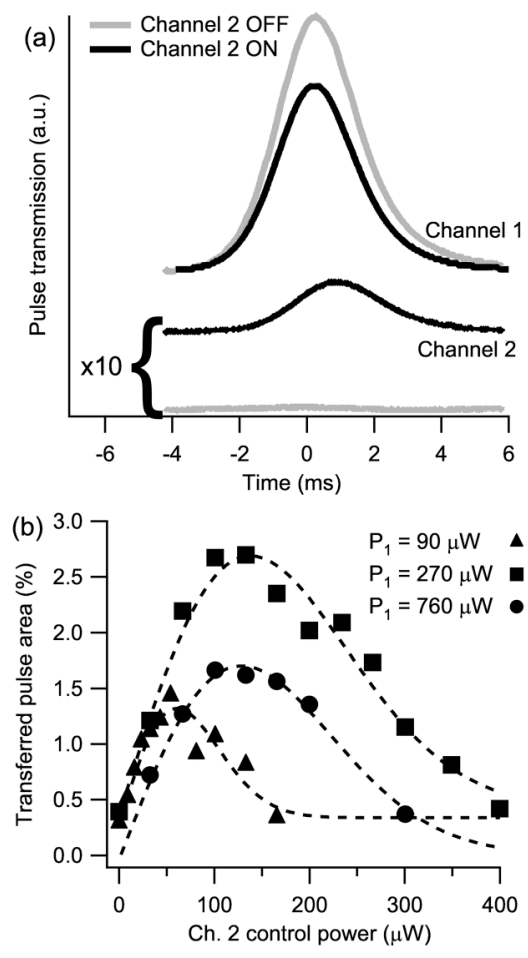

FIG. 4. Pulsed slow light beam splitter. (a) Signal pulses detected from each channel. With Ch. 2 control field on, a slow light pulse is read out in both channels. Input powers: Ch. 1 control $270 \mu \mathrm{W}$, peak signal $9 \mu \mathrm{W}$, Ch. 2 control $150 \mu \mathrm{W}$. (b) Tunable slow light beam splitter. Transferred pulse percentage (area of Ch. 2 pulse normalized to area of Ch. 1 pulse without Ch. 2 light) can be optimized as a function of Ch. 2 power for fixed Ch. 1 powers. Dashed lines added to guide the eye.

Signal transport between beam splitter channels is phase coherent, due to rapid atomic motion in the wall-coated cell. Thus the phase difference between the output signal and control fields in Ch. 2 is determined by the phase difference between the input signal and control fields in Ch. 1. We demonstrated this phase coherence between the two channels by adding half-wave plates at the output of each channel to mix a small part of the corresponding control field into the detected output signal. The resulting interference between the signal and control fields at each PD made the detected amplitudes depend directly on the signal-control phase difference in each channel [17]. Figure 5 shows that the measured output in both channels vary nearly identically as the phase difference between the input signal and control fields in Ch. 1 is scanned by nearly $2 \pi$ using a PZT mounted mirror (see Fig. 2). Note that a conventional beam splitter imparts a $+\pi / 2$ phase shift to signals transferred between channels. This $+\pi / 2$ phase shift can be realized in the slow light beam splitter by applying a two-photon detuning to atoms moving outside the two control field regions, e.g., with an off-resonant laser field. Simulations indicate that such a phase shift will also enable maximally efficient mapping of the input signal pulse into two output pulses while exhibiting standard beam splitter statistics [18]. 


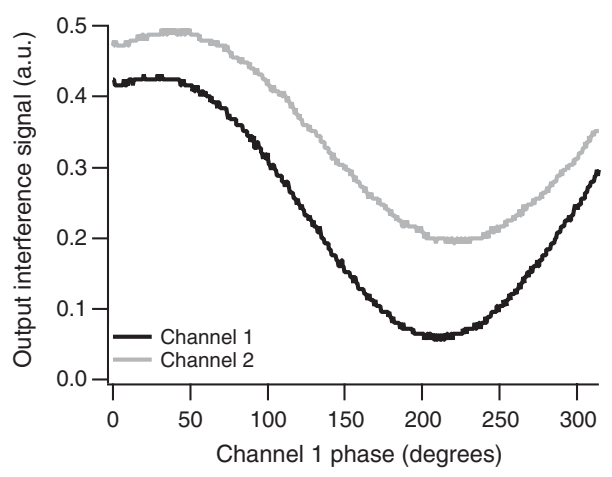

FIG. 5. Demonstration of phase coherent signal transport in the slow light beam splitter. For each channel, a small part of the control field is interfered with the signal field at the photodetectors such that the output signal amplitude is a measure of the phase difference between the signal and control fields. Near identical variation is observed in the two channel outputs as the phase difference between the input signal and control fields in Ch. 1 is scanned by nearly $2 \pi$. Vertical offset in Ch. 1 and Ch. 2 output signals is caused by slightly different control field admixtures. Ch. 1 and Ch. 2 control fields $160 \mu \mathrm{W}$. Input Ch. 1 probe field $13 \mu \mathrm{W}$.

In conclusion, we demonstrated a tunable slow light beam splitter that operates by using rapid transport of atomic coherence in a wall-coated vapor cell. Once optimized, the slow light beam splitter may be used to improve quantum repeater performance and generate nonclassical photon states. This approach may significantly improve the quantum repeater success rate especially for short repeater memory times [19], provided that the beam splitter interferes photons from channels with entanglement in less than the memory storage time of a repeater node. The slow light beam splitter could also increase the efficiency with which maximally-entangled $N$-photon states can be generated from probabilistic single-photon sources (each with generation efficiency $\eta)$. An $M$-port $(M>N / \eta)$ slow light beam splitter could replace a symmetric $N$-port fiber coupler [20]. The increased number of single-photon sources would ensure that more than $N$ photons are generated each cycle, while the dynamic reconfigurability of the beam splitter allows us to activate (and thus interfere) only those channels which contain single photons. For $N=1,2$, this may also have application to generating inputs for quantum computation schemes based on linear optics [21].

The slow light beam splitter may also have applications in classical telecommunications: e.g., as an optical buffer with adjustable multiport output. Here, orthogonal linearly polarized light could be used for the signal and control fields, in order to enhance transport efficiency in both channels via self-rotation induced gain [22]. Potential realizations in other media include ions and atoms in traps, and atoms in hollow core fibers [23] where precise control over the (largely classical) motion of the mediating atomic ensemble would be difficult or impossible. The key result of this work is the demonstration that even particles undergoing random and undirected classical motion can mediate coherent interactions between two or more modes. This work, then, is relevant to a range of fields not only because of the potential uses of the coated-cell beam splitter, but also as a demonstration of a widely applicable concept.

[1] N.S. Ginsberg, S.R. Garner, and L. V. Hau, Nature (London) 445, 623 (2007); B. Wang et al., Phys. Rev. A 72, 043801 (2005); A. S. Zibrov et al., Phys. Rev. Lett. 88, 103601 (2002); C. Schnurr et al., Opt. Lett. 15, 1097 (1990).

[2] T. Wang, M. Kostrun, and S.F. Yelin, Phys. Rev. A 70, 053822 (2004); A. Raczynski, J. Zaremba, and S. Zielinska-Kaniasty, ibid. 75, 013810 (2007).

[3] L.-M. Duan et al., Nature (London) 414, 413 (2001).

[4] H. G. Robinson, E. S. Ensberg, and H. G. Dehmelt, Bull. Am. Phys. Soc. 3, 9 (1958).

[5] H. G. Robinson and C. E. Johnson, IEEE Trans. Instrum. Meas. 32, 198 (1983); R. P. Frueholz, C. H. Volk, and J. C. Camparo, J. Appl. Phys. 54, 5613 (1983).

[6] D. Budker, V. Yashchuk, and M. Zolotorev, Phys. Rev. Lett. 81, 5788 (1998); D. Budker et al., Phys. Rev. A 71, 012903 (2005).

[7] D. Budker et al., Phys. Rev. Lett. 83, 1767 (1999).

[8] M. Klein et al., J. Mod. Opt. 53, 2583 (2006).

[9] B. Julsgaard, A. Kozhekin, and E. S. Polzik, Nature (London) 413, 400 (2001); C. Schori et al., Phys. Rev. Lett. 89, 057903 (2002); B. Julsgaard et al., Nature (London) 432, 482 (2004).

[10] M. Fleischhauer, A. Imamoglu, and J. P. Marangos, Rev. Mod. Phys. 77, 633 (2005).

[11] L. V. Hau et al., Nature (London) 397, 594 (1999).

[12] M. Fleischhauer and M.D. Lukin, Phys. Rev. Lett. 84, 5094 (2000).

[13] In experiments presented here, a maximum of $10 \%$ of the input pulse was present in the medium at any time as the $3 \mathrm{~ms}$ long pulse was delayed by roughly $0.5 \mathrm{~ms}$, limited by available optical depth.

[14] R. H. Dicke, Phys. Rev. 89, 472 (1953); R. P. Frueholz and C. H. Volk, J. Phys. B 18, 4055 (1985).

[15] Y. Xiao et al., Phys. Rev. Lett. 96, 043601 (2006).

[16] M. Hohensee and M. Klein et al. (to be published).

[17] A. Mair et al., Phys. Rev. A 65, 031802(R) (2002); D. F. Phillips et al., Phys. Rev. Lett. 86, 783 (2001).

[18] M. Hohensee et al., R1.00085, DAMOP 2008.

[19] O. A. Collins et al., Phys. Rev. Lett. 98, 060502 (2007).

[20] G. J. Pryde and A. G. White, Phys. Rev. A 68, 052315 (2003).

[21] E. Knill, R. Laflamme, and G. J. Milburn, Nature (London) 409, 46 (2001).

[22] I. Novikova, D. F. Phillips, and R. L. Walsworth, Phys. Rev. Lett. 99, 173604 (2007).

[23] S. Ghosh et al., Phys. Rev. Lett. 94, 093902 (2005). 